\title{
AN ERGODIC THEOREM FOR SEMIGROUPS ${ }^{1}$
}

\author{
M. A. AKCOGLU AND J. CUNSOLO
}

Introduction. The purpose of this paper is to prove a maximal ergodic theorem (Theorem 1) for continuous semigroups of positive contractions. This result is a continuous extension of the maximal ergodic theorem first introduced by Brunel [4] and then generalized in [1] and [2]. The continuous version of Chacon-Ornstein's ratio ergodic theorem which can be proved directly [7], as noted by the referee, is also a corollary of our result. This continuous version of Chacon-Ornstein's theorem was also proved by Berk [3] by different methods.

Definitions and preliminaries. Let $(X, \mathcal{F}, \mu)$ be a $\sigma$-finite measure space, $\left(R^{+}, \mathcal{L}, m\right)$ the Lebesgue measure space on $R^{+}=[0, \infty)$ and $L_{1}=L_{1}(X, \mathcal{F}, \mu)$ the Banach space of equivalence classes of integrable real valued functions on $X$. The elements of $L_{1}$ will be identified in the usual manner and $L_{1}^{+}$will denote the class of nonnegative $L_{1}$ functions.

Let $T=\left\{T_{t}: t \geqq 0\right\}$ denote a semigroup of positive contractions from $L_{1}$ to $L_{1}$, i.e. $T_{0}=I$ the identity operator, $T_{t+8}=T_{t} T_{s}, T_{t} L_{1}^{+} \subset L_{1}^{+}$, $T_{t}$ is a linear operator and

$$
\int_{X}\left|T_{t} f\right| d \mu \leqq \int_{X}|f| d \mu
$$

for all $s, t \geqq 0$ and all $f \in L_{1}$.

We shall assume that $T$ is a strongly continuous semigroup. This means that for every $f \in L_{1}, T_{(\cdot)} f$ is continuous in $L_{1}$ norm on $R^{+}$ which implies that $T(\cdot) f$ is Lebesgue integrable on every finite interval of $R^{+}$. Hence, for every finite interval $[\beta, \gamma]$ there exists a sequence $\left\{f_{n}\right\}$ of $L_{1}$ valued Lebesgue integrable simple functions on $[\beta, \gamma]$ with the following properties:

(i) $\lim _{n m^{\prime} \rightarrow \infty} \int_{[\beta, \gamma]}\left\|f_{n}(t)-f_{m^{\prime}}(t)\right\| m(d t)=0$ and

(ii) for every $\epsilon>0$

$$
\lim _{n \rightarrow \infty} m\left\{t \mid t \in[\beta, \gamma], \quad\left\|T_{t} f-f_{n}(t)\right\| \geqq \epsilon\right\}=0
$$

1 This research is supported in part by N.R.C. Grant A3974.

Received by the editors November 13, 1968. 
and we define

$$
\int_{\beta}^{\gamma} T_{t} f m(d t)=\lim _{n \rightarrow \infty} \int_{\beta}^{\gamma} f_{n}(t) m(d t) .
$$

To give meaning to $\lim \sup _{\alpha \rightarrow \infty} \int_{0}^{\alpha} T_{t} f m(d t) f \in L_{1}$, we must give a more explicit definition of $\int_{\beta}^{\alpha} T_{t} f(x) m(d t) x \in X, 0 \leqq \beta \leqq \gamma$. Consider $(P, \Sigma, \rho)$ the product measure space of $\left(R^{+}, \mathcal{L}, m\right)$ and $(X, \mathcal{F}, \mu)$. Since, by assumption, $T_{(\cdot)} f$ is Lebesgue integrable for every $f \in L_{1}$, then for each $f \in L_{1}[6$, VII. 7$]$ there exists a real valued $\rho$ measurable function $g(\cdot, \cdot)$ on $P$, uniquely determined up to $\rho$ measure zero, such that:

(i) $g(t, \cdot)=T_{t} f(\cdot)$ for a.e. $t \in R^{+}$and

(ii) there exists a $\mu$ null set $N(f)$ which is independent of $t$ but may depend on $f$ with the property that $g(\cdot, x)$ is Lebesgue integrable on every finite interval of $R^{+}$for every $x$ not in $N(f)$ and $\int_{\beta}^{\gamma} g(t, x) m(d t)$ as a function of $x$ equals $\int_{\beta}^{\gamma} T_{t} f m(d t)$ for every $x$ not in $N(f)$.

Hence the meaning of $\int_{\beta}^{\gamma} T_{t} f(x) m(d t)$ is obtained from $\int_{\beta}^{\gamma} g(t, x) m(d t)$ for every $x$ not in $N(f)$.

Since $g(\cdot, x)$ is Lebesgue integrable on every finite interval of $R^{+}$ for every $x \notin N(f)$ then $\int_{0}^{\alpha} g(t, x) m(d t)$ is continuous in $\alpha$ on $(0, \infty)$. Let $R_{r}^{+}$denote the countable set of positive rationals; then from the continuity of $\int_{0}^{\alpha} g(t, x) m(d t)$ it follows that

$$
\text { (i) } \inf _{\alpha \geqq 0} \sup _{\alpha^{\prime} \geqq \alpha} \int_{0}^{\alpha^{\prime}} g(t, x) m(d t)=\inf _{r \in R_{r}^{+}} \sup _{\substack{r^{\prime} \geqq r \\ r \in R_{r}^{+}}} \int_{0}^{r^{\prime}} g(t, x) m(d t)
$$

for $x \notin N(f)$.

Meaning is given to $\lim \sup _{\alpha \rightarrow \infty} \int_{0}^{\alpha} T_{t} f m(d t)$ by

$$
\begin{array}{r}
\left(\limsup _{\alpha \rightarrow \infty} \int_{0}^{\alpha} T_{t} f m(d t)\right)(x)= \\
=\inf _{\alpha \geqq o} \sup _{\alpha^{\prime} \geqq \alpha} \int_{0}^{\alpha^{\prime}} g(t, x) m(d t) \\
=\left(\limsup _{r \rightarrow \infty} \int_{0}^{r} T_{t} f m(d t)\right)(x) \\
\text { by (i) for } x \in N(f)
\end{array}
$$

where $\lim$ sup is taken along the positive reals and positive rationals respectively. Thus $\lim \sup _{\alpha \rightarrow \infty} \int_{0}^{\alpha} T_{t} f m(d t)$ is a real $\mu$ measurable function defined a.e. on $X$.

We shall use the following abbreviations: 


$$
\begin{gathered}
S_{\beta}^{\gamma}(f)=\int_{\beta}^{\gamma} T_{t} f m(d t), \quad S_{\beta}^{\gamma}(f ; x)=\int_{\beta}^{\gamma} T_{t} f(x) m(d t), \\
\chi_{E} S_{\beta}^{\gamma}(f)=\int_{\beta}^{\gamma} \chi_{E} T_{t} f m(d t)
\end{gathered}
$$

for $E \in \mathcal{F}$ where $\chi_{E}$ is the characteristic function of $E$ and $0 \leqq \beta \leqq \gamma$; if $\beta=0$ we shall write $S^{\gamma}(f)$ and $S^{\gamma}(f: x)$ respectively.

Definition 1. Let $\tau=\left\{0, t_{1}, t_{2}, \cdots, t_{n}\right\}$ denote a finite partition of $R^{+}$into intervals such that $t_{i-1}<t_{i}$ for $1 \leqq i \leqq n=n(\tau)$ and $t_{0}=0$ and define $\tau_{i}=t_{i}-t_{i-1}, \tau_{0}=0$ for $1 \leqq i \leqq n$. Denote by $\odot$ the class of all such finite partitions $\tau$ on $R^{+}$.

Definition 2. For any $\tau \in \mathcal{P}, f \in L_{1}^{+}$and $E \in \mathcal{F}$ we define the following finite sequence of $L_{1}^{+}$functions $\left\{a_{i}(\tau)\right\}, i=1,2, \cdots, n(\tau)$ by

$$
\begin{aligned}
a_{0}(\tau) & =\chi_{E} f \\
a_{m}(\tau) & =\chi_{E}\left\{T_{\tau_{m}} f-\sum_{\nu=1}^{m} T_{\tau_{m}-\tau_{m-\nu}} a_{m_{-v}}(\tau)\right\}, \quad 1 \leqq m \leqq n(\tau) .
\end{aligned}
$$

It can be shown by induction that

$$
a_{m}(\tau)=\chi_{E} T_{\tau_{m}} \chi_{E^{c}} T_{\tau_{m-1}} \chi_{E^{c}} \cdots \chi_{E^{c}} T_{\tau_{1}} \chi_{E}^{c} f, 1 \leqq m \leqq n(\tau) .
$$

Definition 3. We define for $E \in F, f \in L_{1}^{+}$and $\tau \in \mathcal{P}$

$$
\begin{aligned}
& \Omega_{E}^{\tau}(f)=\sum_{\nu=0}^{n(\tau)} \int a_{\nu}(\tau) d \mu, \\
& \Omega_{E}(f)=\sup _{\tau \in \mathcal{P}} \Omega_{E}^{\tau}(f)
\end{aligned}
$$

and if $g \in L_{1}$ then $g=g^{+}-g^{-}, g^{+}$and $g^{-} \in L_{1}^{+}$and we define

$$
\Omega_{E}(g)=\Omega_{E}\left(g^{+}\right)-\Omega_{E}\left(g^{-}\right) .
$$

It can be seen from the definitions that $\Omega_{E}(\alpha f)=\alpha \Omega_{E}(f)$ for any $\alpha \geqq 0, \Omega_{E}(f) \leqq\|f\|$ for any $f \in L^{+}, \Omega_{E}(f)=0$ if and only if $S^{\infty}(f)=0$ a.e. on $E$ and if the elements of $\tau$ are contained in $\tau^{\prime}\left(\tau \subset \tau^{\prime}\right)$ and $f \in L_{1}^{+}$ then $\Omega_{E}^{\tau}(f) \leqq \Omega_{E}^{\tau^{\prime}}(f)$.

A maximal ergodic theorem. The following theorem is an extension of the maximal ergodic theorem in [1] to the continuous case. To prove this theorem we shall use discrete approximations. 
THEOREM 1. Let $E$ be any subset of $\mathcal{F}$ and $f$ be any $L_{1}$ function. Then $\lim \sup _{\alpha \rightarrow \infty} S^{\alpha}(f)>0$ a.e. on $E$ implies that $\Omega_{E}(f) \geqq 0$ or equivalently $\Omega_{E}\left(f^{+}\right) \geqq \Omega_{E}\left(f^{-}\right)$where $f=f^{+}-f^{-}, f^{+}$and $f^{-} \in L_{1}^{+}$.

For this proof we need the following lemmas:

Lemma 1. For any $f \in L_{1}^{+}$and $\epsilon>0$ there exists a rational $r>0$ and $a$ positive integer $n$ such that

$$
\left|\Omega_{E}(f)-\Omega_{E}^{\tau}(f)\right|<\epsilon \text { where } \tau=\{0, r, 2 r, \cdots, n r\} .
$$

Proof. Choose $\epsilon>0$; then there exists a partition $\tau^{0} \in \mathcal{P}, \tau^{0}$ $=\left\{0, t_{1}, t_{2}, \cdots, t_{n}\right\}$ such that

$$
\left|\Omega_{E}(f)-\Omega_{E}^{\tau^{0}}(f)\right|<\epsilon / 2
$$

We now construct a partition $\tau^{N}=\left\{0, r_{1}, r_{2}, \cdots, r_{N}\right\}$ where $r_{1}, r_{2}$, $\cdots, r_{n}$ are positive rationals which will be determined by induction and $\tau^{N}$ has the property that $\left|\Omega_{E 1}^{\tau^{0}}(f)-\Omega_{E}^{\tau^{N}}(f)\right|<\epsilon / 2$. Let $\tau^{m}, 1 \leqq m \leqq N$, denote the partition $\left\{0, r_{1}, r_{2}, \cdots, r_{m-1}, r_{m}, t_{m+1}, \cdots, t_{N}\right\}$.

For $t, t_{1} \leqq t<t_{2}$ we have the following inequalities:

(i) $\left|\int_{E} T_{t_{1}} \chi_{E^{c}} f d \mu-\int_{E} T_{t} \chi_{E^{c}} f d \mu\right| \leqq\left\|T_{t_{1}} \chi_{E^{c}} f-T_{t} \chi_{E^{c}} f\right\|$

(ii) $\left|\int_{E} T_{t_{2}-t_{1}} \chi_{E^{c}} T_{t_{1}} \chi_{E^{c}} f d \mu-\int_{E} T_{t_{2}-t} \chi_{E^{c}} T_{t} \chi_{E^{c}} f d \mu\right|$

$\leqq\left\|T_{t_{2}-t}\left(T_{t-t_{1}} \chi_{E^{c}} T_{t_{1}} \chi_{E^{c}} f-\chi_{E^{c}} T_{t-t_{1}} T_{t_{1}} \chi_{E^{c}} f\right)\right\|$

$\leqq\left\|T_{t-t_{1}} \chi_{E^{c}} T_{t_{1}} \chi_{E^{c}} f-\chi_{E^{c}} T_{t_{1}} \chi_{E^{c}} f\right\|+\left\|T_{t_{1}} \chi_{E^{c}} f-T_{t-t_{1}}\left(T_{t_{1}} \chi_{E^{c}} f\right)\right\|$

(iii) $\left|\int a_{m}\left(\tau^{0}\right) d \mu-\int T_{t_{m}-t_{m-1}} \chi_{E^{c}} \cdots \chi_{E^{c}} T_{t_{2}-t} \chi_{E^{c}} T_{t} \chi_{E^{c}} f d \mu\right|$ $\leqq\left\|T_{t_{2}-t_{1}} \chi_{E^{c}} T_{t_{1}} \chi_{E^{c}} f-T_{t_{2}-t} \chi_{E^{c}} T_{t} \chi_{E^{c}} f\right\| \quad$ for $3 \leqq m \leqq N$.

By (i), (ii) and (iii) and the continuity of $T_{(\cdot) f}$ in $L_{1}$ norm on $[0, \infty)$ we can choose a rational $r_{1}, t_{1} \leqq r_{1}<t_{2}$ such that the right-hand sides of the inequalities (i), (ii) (and hence (iii)) are each less than or equal $\epsilon / 2 N$.

Define $\tau^{1}=\left\{0, r_{1}, t_{2}, \cdots, t_{N}\right\}$. Then by our choice of $r_{1}$ we have

$$
\left|\Omega_{E}^{\tau^{0}}(f)-\Omega_{E}^{\tau^{1}}(f)\right| \leqq \sum_{\nu=1}\left|\int a_{\nu}\left(\tau^{0}\right) d \mu-\int a_{\nu}\left(\tau^{1}\right) d \mu\right|<\epsilon / 2 N
$$

In a similar fashion we can find rationals $r_{2}, r_{3}, \cdots, r_{N}, t_{i} \leqq r_{i}<t_{i+1}$, $i=2,3, \cdots N-1$ and $r_{N} \geqq t_{N}$, such that the corresponding partitions $\tau^{2}, \tau^{3}, \cdots \tau^{N}$ have the property that

$$
\left|\Omega_{E}^{\tau^{i-1}}(f)-\Omega_{E}^{\tau^{i}}(f)\right|<\epsilon / 2 N \quad i=2,3, \cdots, N .
$$


Hence

$$
\left|\Omega_{E}(f)-\Omega_{E}^{\tau^{N}}(f)\right| \leqq\left|\Omega_{E}(f)-\Omega_{E}^{\tau^{0}}(f)\right|+\sum_{\nu=1}^{N}\left|\Omega_{E}^{\tau^{i-1}}(f)-\Omega_{E}^{\tau^{i}}(f)\right|<\epsilon .
$$

Let $r$ denote the reciprocal of the least common denominator of $\left\{r_{1}, r_{2}, \cdots, r_{N}\right\}$ then $r_{i}=m_{i} r$ where $m_{i}$ is some positive integer for $i=1,2, \cdots, N$. Putting $n=\sum_{i=1}^{N} m_{i}$, we define the partition $\tau$ $=\{0, r, 2 r, \cdots, n r\}$. Since $f \in L_{1}^{+}$and $\tau^{N} \subset \tau$ then

$$
0 \leqq \Omega_{E}^{\tau^{N}}(f) \leqq \Omega_{E}^{\tau}(f) \leqq \Omega_{E}(f)
$$

which implies that $\left|\Omega_{E}(f)-\Omega_{E}^{\tau}(f)\right|<\epsilon$.

Note that since $T$ is a strongly continuous semigroup of positive contractions then for $A \in \mathcal{F}$ and $f \in L_{1}$, the function $\chi_{A} T_{(\cdot)} f$ is continuous on $[0, \infty)$ with respect to $L_{1}$ norm. Hence we can approximate the function $\chi_{A} S^{\alpha}(f)$ in $L_{1}$ norm by finite sums of the form $\sum_{i=1}^{N} \chi_{A} T_{t_{i}} f \triangle t_{i}$ for $\alpha>0$.

Lemma 2. Let $(X, \mathcal{F}, \mu)$ be a $\sigma$-finite measure space and $T=\left\{T_{t}: t \geqq 0\right\}$ a strongly continuous semigroup of positive contractions. Consider a set $E \in \mathcal{F}$ and $f \in L_{1}$ where $f=f^{+}-f^{-}\left(f^{+}, f^{-} \in L_{1}^{+}\right)$and $\chi_{E} f^{-}=f^{-}$. If $\lim \sup _{\alpha \rightarrow \infty} S^{\alpha}(f)>0$ a.e. on $E$ then $\Omega_{E}(f) \geqq 0$.

Proof. Choose $\epsilon>0$. The continuity of $S^{\alpha}(f ; x), x \notin N(f)$ and $\lim \sup _{\alpha \rightarrow \infty} S^{\alpha}(f)>0$ a.e. on $E$ imply that for a.e. $x$ in $E$ there exists a rational $r=r(x)$ such that $S r(f ; x)>0$. Consider the indexed positive rationals $r_{1}, r_{2}, \cdots$ and define

$$
E_{i}=\left\{x \mid S^{r_{i}}(f ; x)>0, x \in E\right\} .
$$

It follows that $E_{i} \in \mathcal{F}$ for $i=1,2, \cdots$ and $\mu\left(\cup_{i=1}^{\infty} E_{i}\right)=\mu(E)$.

We choose $i$ sufficiently large, say $i=k$, such that $\int_{E-\cup_{i-1}^{k} E_{i}}^{k} f d \mu$ $<\epsilon / 4$ (i).

By the construction of $E_{i}, i=1,2, \cdots k, S^{r}(f)>0$ on $E_{i}$ hence there exists a measurable subset $C_{i}$ of $E_{i}$ and a $\gamma_{i}>0$ such that $\chi_{C_{i}} S_{i}(f) \geqq \gamma_{i}$ and $\int_{E_{i}-C_{i}} f^{-} d \mu<\epsilon / 4 k$. Thus

(ii) $\bigcup_{i=1}^{k} E_{i}=\bigcup_{i=1}^{k} C_{i} \cup \bigcup_{i=1}^{k}\left(E_{i}-C_{i}\right)$

and

(iii) $\quad \int_{i=1}^{k} \bigcup_{\left(E_{i}-C_{i}\right)} f^{-} d \mu \leqq \sum_{i=1}^{k} \int_{E_{i}-C_{i}} f^{-} d \mu<\epsilon / 4$. 
For each $C_{i}, i=1,2, \cdots k$ define

$$
\begin{gathered}
B_{i}^{n}=\left\{x \in C_{i} \mid \frac{r_{i}}{n} \sum_{j=0}^{n-1} \chi_{C_{i}} T_{r_{i} / n}^{j} f(x) \leqq 0\right\} \quad \text { and } \\
A_{i}^{n}=C_{i}-B_{i}^{n}=\left\{x \in C_{i} \mid \frac{r_{i}}{n} \sum_{j=0}^{n-1} \chi_{C_{i}} T_{r_{i} / n}^{j} f(x)>0\right\} .
\end{gathered}
$$

Clearly $B_{i}^{n} \in \mathcal{F}$ for all $n>0$. Since

$$
\begin{aligned}
& \lim _{n \rightarrow \infty}\left\|\chi_{C_{i}} S^{r}{ }^{r}(f)-\frac{r_{i}}{n} \sum_{j=0}^{n-1} \chi_{C_{i}} T_{r_{i} / n} f\right\|=0 \\
& 0 \leqq\left\|\chi_{B_{i}{ }^{n} S^{r}}{ }^{i}(f)\right\| \leqq\left\|\chi_{C_{i}} S^{r} i(f)-\frac{r_{i}}{n} \sum_{j=0}^{n-1} \chi_{C_{i}} T_{r_{i} / n}^{j}\right\| \text { for } n>0
\end{aligned}
$$

and

$$
0 \leqq \gamma_{i} \mu\left(B_{i}^{n}\right) \leqq \int_{B_{i}^{n}} S^{r_{i}}(f) d \mu=\left\|\chi_{B_{i}^{n}} S^{r_{i}}(f)\right\| \text { for all } n>0
$$

then for any $\delta>0$ there exists an integer $M_{i}=M_{i}(\delta, i)$ with the property that $\mu\left(B_{i}^{M_{i}}\right) \leqq \delta, i=1,2, \cdots, k$. Since, as a set function, $\int f^{-} d \mu$ is absolutely continuous with respect to $\mu$ then for $\epsilon / 4 k>0$ there exists a $\delta=\delta(\epsilon / 4 k)>0$ such that if $F \in \mathcal{F}$ and $\mu(F) \leqq \delta$ then $\int_{m} f^{-} d \mu \leqq \epsilon / 4 k$. Hence for each $i, i=1,2, \cdots, k$ there exists an integer $M_{i}=M_{i}(\delta, i)$ such that

$$
\int_{\substack{M_{i} \\ M_{i}}} f^{-} d \mu \leqq \epsilon / 4 k, \quad i=1,2, \cdots, k
$$

Thus

$$
\text { (iv) } \quad \bigcup_{i=1}^{k} C_{i}=\bigcup_{i=1}^{k} A_{i}^{M_{i}} \cup \bigcup_{i=1}^{k} B_{i}^{M_{i}}
$$

and

$$
\text { (v) } \quad \int_{i=1}^{k} B_{j}^{M_{i}} f^{-} d \mu \leqq \sum_{i=1}^{k} \int_{B_{i}^{M_{i}}} f^{-} d \mu \leqq \epsilon / 4 .
$$

By putting $A=\bigcup_{i=1}^{k} A_{i}^{M_{i}}$ and using (i), (ii), (iii), (iv), (v) we have

$$
\int_{E} f^{-} d u<\int_{A} f^{-} d \mu+3 \epsilon / 4 .
$$


Let $r$ be the reciprocal of the least common denominator of $\left\{r_{i} / M, i=1,2, \cdots, k\right\}$ where $M=\max \left\{M_{i}, i=1,2, \cdots, k\right\}$ then by the construction of $A$ and $r$ it follows that

$$
\sup _{n} \sum_{j=0}^{n} \chi_{A} T_{r}^{j} f>0 \quad \text { a.e. on } A \text {. }
$$

This together with the first part of the proof of Theorem 1 [2] implies that

$$
\Omega_{A}^{r}\left(f^{+}\right) \geqq \int_{A} f^{-} d \mu
$$

where

$$
\begin{aligned}
\Omega_{A}^{r}\left(f^{+}\right) & =\sum_{\nu=0}^{\infty} \int a_{\nu} d \mu \quad a_{0}=\chi_{A} f \text { and } \\
a_{n} & =\chi_{A}\left(T_{r} \chi_{A} c\right)^{n} f \text { for } n \geqq 1 .
\end{aligned}
$$

There exists an integer $N$ such that for the partition $\tau$ $=\{0, r, 2 r, \cdots N r\}$,

$$
\Omega_{A}^{\tau}\left(f^{+}\right)>\Omega_{A}^{r}\left(f^{+}\right)-\epsilon / 4 .
$$

Hence

$$
\begin{aligned}
\Omega_{E}\left(f^{+}\right) \geqq \Omega_{E}^{\tau}\left(f^{+}\right) \geqq \Omega_{A}^{\tau}\left(f^{+}\right)>\Omega_{A}^{r}\left(f^{+}\right)-\epsilon / 4 & \geqq \int_{A} \bar{f} d \mu-\epsilon / 4 \\
& >\int_{E} f^{-} d \mu-\epsilon=\Omega_{E}\left(f^{-}\right)-\epsilon .
\end{aligned}
$$

Therefore, $\Omega_{E}\left(f^{+}\right)-\Omega_{E}\left(f^{-}\right)>-\epsilon$ for any $\epsilon>0$ which implies $\Omega_{E}\left(f^{+}\right)$ $\geqq \Omega_{E}\left(f^{-}\right)$which completes the proof.

Lemma 3. Let $f$ and $g \in L_{1}^{+}$, then for any $\beta>0$

$$
\lim _{\alpha \rightarrow \infty} \frac{S_{\alpha}^{\alpha+\beta}(g)}{S^{\alpha}(f)}=0 \quad \text { a.e. on }\left\{x \in X \mid S^{\infty}(f ; x)>0\right\} .
$$

Proof. Choose $\beta>0$. For any real number $\alpha \geqq \beta$ there exists an integer $n=n(\alpha, \beta)$ with the following properties: $n \beta \leqq \alpha$ and $\alpha=\alpha_{0}+\beta$ where $\alpha_{0}=\alpha_{0}(\alpha, \beta)$ and $0 \leqq \alpha_{0}<\beta$.

Hence $S_{\alpha}^{\alpha+\beta}(g)=T_{\beta}^{n} S_{\alpha_{0}}^{\alpha_{0}+\beta}(g) \leqq T_{\beta}^{n} S^{2 \beta}(g)$ a.e. on $X$ and $S^{\alpha}(f) \geqq S^{n \beta}(f)$ $=\sum_{\nu=0}^{n} T_{\beta}^{\nu} S^{\beta}(f)$ a.e. on $X$. This yields 


$$
0 \leqq \frac{S_{\alpha}^{\alpha+\beta}(g)}{S^{\alpha}(f)} \leqq \frac{T_{\beta}^{n} S^{2 \beta}(g)}{\sum_{\nu=0}^{n} T_{\beta}^{\nu} S^{\beta}(f)} \text { a.e. on the support of } \sum_{\nu=0}^{n} T_{\beta}^{\nu} S^{\beta}(f) .
$$

Since

$$
\lim _{n \rightarrow \infty} \frac{T_{\beta}^{n} S^{2 \beta}(g)}{\sum_{\nu=0}^{n} T_{\beta}^{\nu} S^{\beta}(f)}=0
$$

a.e. on

$$
\left\{x \in X \mid\left(\sum_{\nu=0}^{\infty} T_{\beta}^{\nu} S^{\beta}(f)\right)(x)>0\right\}=\left\{x \in X \mid S^{\infty}(f ; x)>0\right\}=A
$$

then

$$
\lim _{\alpha \rightarrow \infty} \frac{S_{\alpha}^{\alpha+\beta}(g)}{S^{\alpha}(f)}=0 \quad \text { a.e. on } A
$$

which completes the proof.

Lemma 4. Given $f$ and $g \in L_{1}^{+}$, then for any real number $\beta>0$ and integer $n \geqq 0$ define

$$
\tilde{g}=\chi_{E} g+\chi_{E} T_{\beta} \chi_{E^{c}} g+\chi_{E} T_{\beta} \chi_{E^{c}} T_{\beta} \chi_{E^{c}} g+\cdots+\chi_{E}\left(T_{\beta} \chi_{E^{c}}\right)^{n} g .
$$

If, for any $0<\lambda<1$ and $S^{\infty}(g)>0$ a.e. on $E$,

$$
\begin{array}{ll}
\lim \sup _{\alpha \rightarrow \infty} S^{\alpha}(f-g)>0 & \text { a.e. on } E \text { then } \\
\lim \sup _{\alpha \rightarrow \infty} S^{\alpha}(f-\lambda \tilde{g})>0 & \text { a.e. on } E .
\end{array}
$$

Proof. Choose a real number $\beta>0$ and an integer $n \geqq 0$ and let $a_{0}=\chi_{E} g$ and $a_{\nu}=\chi_{E}\left(T_{\beta} \chi_{E^{c}}\right)^{\nu} g=\chi_{E}\left(T_{\beta}^{\nu} g-\sum_{j=1}^{\nu} T_{\beta}^{j} a_{\nu-j}\right)$ for $\nu=1,2$, $\cdots, n$; define $\tilde{g}=\sum_{\nu=0}^{n} a_{\nu}$.

For any $\alpha>0$ we have

(i) $\quad S^{\alpha}(f-\lambda \tilde{g})=S^{\alpha}(f)-\lambda\left[\sum_{\nu=0}^{n-1} S^{\alpha}\left(a_{\nu}\right)-S^{\alpha}\left(a_{n}\right)\right]$.

Since we are restricting ourselves to the set $E$ nothing is lost by writing

$$
\begin{aligned}
a_{n}= & T_{\beta}^{n} g-\sum_{j=1}^{n} T_{\beta}^{j} a_{n-j}=T_{\beta}\left[T_{\beta}^{n-1} g-\sum_{j=1}^{n-1} T_{\beta}^{j} a_{n-1-j}-a_{n-1}\right] \\
& \cdot S^{\alpha}\left(a_{n}\right)=S_{\beta}^{\alpha+\beta}\left(T_{\beta}^{n-1} g-\sum_{j=1}^{n-1} T_{\beta}^{j} a_{n-1-j}-a_{n-1}\right) \\
\leqq & S^{\alpha+\beta}\left(T_{\beta}^{n-1} g-\sum_{j=1}^{n-1} T_{\beta}^{j} a_{n-1-j}-a_{n-1}\right) .
\end{aligned}
$$


Substitution in (i) and simple calculations yield

$$
S^{\alpha}(f-\lambda \tilde{g}) \geqq S^{\alpha}(f)-\lambda\left[\sum_{\nu=0}^{n-2} S^{\alpha}\left(a_{v}\right)-S^{\alpha+\beta}\left(T_{\beta}^{n-1} g-\sum_{j=1}^{n-1} T_{\beta}^{\prime} a_{n-1-j}\right)\right] .
$$

By rer eating this process for $a_{n-1}, a_{n-2}, \cdots a_{1}$, we have

$$
S^{\alpha}(f-\lambda \tilde{g}) \geqq S^{\alpha}(f)-\lambda S^{\alpha}(g)-\lambda S_{\alpha}^{\alpha+n \beta}(g) \text { for every } \alpha \geqq 0 \text { a.e. on } E \text {, }
$$

which implies that

$$
\limsup _{\alpha \rightarrow \infty} S^{\alpha}(f-\lambda \tilde{g}) \geqq \limsup _{\alpha \rightarrow \infty}\left[S^{\alpha}(f)-\lambda S^{\alpha}(g)-\lambda S_{\alpha}^{\alpha+n \beta}(g)\right] .
$$

Since

$$
\lim _{\alpha \rightarrow \infty} \lambda \frac{S_{\alpha}^{\alpha+n \beta}(g)}{S^{\alpha}(g)}=0 \quad \text { a.e. on }\left\{x \mid S^{\infty}(g ; x)>0\right\}
$$

by Lemma 3 and

$$
\limsup _{\alpha \rightarrow \infty} S^{\alpha}(f-g)>0 \quad \text { a.e. on } E
$$

then it follows that

$$
\limsup _{\alpha \rightarrow \infty}\left[S^{\alpha}(f)-\lambda S^{\alpha}(g)-\lambda S_{\alpha}^{\alpha+\beta}(g)\right]>0
$$

a.e. on $E^{\prime}=E \cap\left\{x \mid S^{\infty}(g ; x)>0\right\}$ and that $E-E^{\prime}$ is a set of $\mu$ measure zero. Thus $\lim \sup _{\alpha \rightarrow \infty} S^{\alpha}(f-\lambda \tilde{g})>0$ a.e. on $E$ which completes the proof.

Proof of Theorem 1. Choose any $\epsilon>0$. By Lemma 1 there exists a rational $r>0$ and an integer $n$ such that on $E^{\prime}=E \cap\left\{x \mid S^{\infty}\left(f^{-} ; x\right)>0\right\}$

$$
\left|\Omega_{E^{\prime}}\left(f^{\top}\right)-\Omega_{E^{\prime}}^{\tau}(\bar{f})\right|<\epsilon \text { where } \tau=\{0, r, 2 r, \cdots, n r\}
$$

and $\Omega_{E^{\prime}}^{r}\left(f^{-}\right)=\sum_{\nu=0}^{n} \int a_{\nu}(\tau) d \mu$ where $a_{0}(\tau)=\chi_{E^{\prime}} f^{-}$and $a_{\nu}=\chi_{E^{\prime}}\left(T_{r} \chi_{E^{\prime c}}\right)^{\nu f^{-}}$ for $1 \leqq \nu \leqq n$.

Define $g=\sum_{\nu=0}^{n} a_{\nu}(\tau)$ then it follows from Lemmas 4 and 2 respectively that, for any $0<\lambda<1, \lim \sup _{\alpha \rightarrow \infty} S^{\alpha}\left(f^{+}-\lambda g\right)>0$ a.e. on $E^{\prime}$ and $\Omega_{E^{\prime}}\left(f^{+}\right) \geqq \lambda \Omega_{E^{\prime}}(g)$ which yields that $\Omega_{E^{\prime}}(f) \geqq \Omega_{E^{\prime}}(g)$. Hence $\Omega_{E^{\prime}}\left(f^{+}\right)$ $\geqq \Omega_{E^{\prime}}(g)=\Omega_{E^{\prime}}^{\tau}\left(f^{-}\right)>\Omega_{E^{\prime}}\left(f^{-}\right)-\epsilon$ which yields $\Omega_{E^{\prime}}\left(f^{+}\right) \geqq \Omega_{E^{\prime}}\left(f^{-}\right)$. Since $S^{\infty}\left(f^{-}\right)=0$ a.e. on $E-E^{\prime}$ then $\Omega_{E-E^{\prime}}\left(f^{-}\right)=0$. Hence

$$
\Omega_{E}\left(f^{+}\right) \geqq \Omega_{E^{\prime}}\left(f^{+}\right) \geqq \Omega_{E^{\prime}}\left(f^{-}\right)+\Omega_{E-E^{\prime}}\left(f^{-}\right) \geqq \Omega_{E}\left(f^{-}\right)
$$

which completes the proof. 
A ratio ergodic theorem. The following theorem which extends the ratio ergodic theorem of Chacon and Ornstein to the continuous case is a direct consequence of Theorem 1 and its proof is identical to the proof of Theorem 2 in [2] so only an outline of the proof will be given.

Theorem 2. Let $f \in L_{1}^{+}, g \in L_{1}$ and $E \in F$, then $\lim _{\alpha \rightarrow \infty} S^{\alpha}(g) / S^{\alpha}(f)$ exists (and is finite) a.e. on $E^{\prime}=E \cap\left\{x \in X \mid S^{\infty}(f ; x)>0\right\}$.

Proof. If $\lim \sup _{\alpha \rightarrow \infty} S^{\alpha}(g) / S^{\alpha}(f)$ is infinite a.e. on $E^{\prime}$ then by Theorem 1,

$\Omega_{B}(g) \geqq \gamma \Omega_{B}(f)$ for some nonnegligible subset $B$ of $E$ and for all $\gamma \geqq 0$ which is a contradiction since $\Omega_{B}(g)<\infty$ and $\Omega_{B}(f)>0$.

If $\lim _{\alpha \rightarrow \infty} S^{\alpha}(g) / S^{\alpha}(f)$ does not exist a.e. on $E^{\prime}$ then there exists a nonnegligible subset $G$ of $E^{\prime}$, two numbers $\gamma<\beta$ and a nonnegligible subset $G^{\prime}$ of $G$ with property that

$$
\liminf _{\alpha \rightarrow \infty} \frac{S^{\alpha}(g)}{S^{\alpha}(f)} \leqq \gamma<\beta \leqq \limsup _{\alpha \rightarrow \infty} \frac{S^{\alpha}(g)}{S^{\alpha}(f)} \text { a.e. on } G^{\prime} .
$$

This implies, by Theorem 1 , that $\gamma \Omega_{G^{\prime}}(f) \geqq \Omega_{G^{\prime}}(g)$ and $\Omega_{G^{\prime}}(g) \geqq \beta \Omega_{G^{\prime}}(f)$ which is a contradiction.

\section{REFERENCES}

1. M. A. Akcoglu, An ergodic lemma, Proc. Amer. Math. Soc. 16 (1965), 388-392. MR 31 \#3573.

2. - Pointwise ergodic theorems, Trans. Amer. Math. Soc. 125 (1966), 296309. MR 37 \#6434.

3. K. N. Berk, Ergodic theory with recurrent weights, Am. Math. Statist. 39 (1968), 1107-1114. MR 37 \#5359.

4. A. Brunel, Sur un lemme ergodique voisin du lemme de $E$. Hopf et sur une de ses applications, C. R. Acad. Sci. Paris 256 (1963), 5481-5484. MR 27 \#2608.

5. R. V. Chacon and D. S. Ornstein, A general ergodic theorem, Illinois J. Math. 4 (1960), 153-160. MR 22 \#1822.

6. N. Dunford and J. T. Schwartz, Linear Operators. Part I: General theory, Pure and Appl. Math., vol. 7, Interscience, New York, 1958. MR 22 \#8302.

7. H. Fong, A continuous analogue of the Chacon-Ornstein theorem (to appear).

UNIVERSITY OF TORONTO 